Draft VERSION JunE 1, 2018

Preprint typeset using $\mathrm{LAT}_{\mathrm{E} X}$ style emulateapj v. 4/12/04

\title{
SWIFT J1644+57: AN ULTRA-LUMINOUS X-RAY EVENT
}

\author{
Aristotle Socrates \\ Institute for Advanced Study, Einstein Drive, Princeton, NJ 08540: socrates@ias.edu \\ Draft version June 1, 2018
}

\begin{abstract}
The photon spectral energy distribution of the powerful transient Sw J1644+57 resembles those of the brightest Ultra-Luminous X-ray sources (ULXs). The transient nature of Sw J1644+57 is likely the result of a tidal disruption of a star by a super-massive black hole. The stellar disk generates accretion power at super-Eddington rates and the observational properties of Sw J1644+57 indicate - in analogy with ULXs - that the accretion flow maintains a high level of radiative efficiency with a corresponding super-Eddington luminosity. Due to its similarity to ULXs, this powerful transient may be thought of as an Ultra-Luminous X-ray event (ULX-E). Observational tests for this ULX-E model are proposed as well.
\end{abstract}

Subject headings: black hole accretion

\section{OVERVIEW AND OBSERVATIONAL FACTS FOR SW J1644+57}

A powerful broad-band source, Sw J1644+57 discovered by the Swift space observatory on March 28, 2011 represents genuinely new phenomena. The combination of its flux, fluence, spectra and duration are unique and clearly, the excitement that surrounds its discovery shows the potential of wide-field time domain astronomy.

A compilation of the observed properties of $\mathrm{Sw}$ $1644+57$ can be found in Levan et al. (2011) and Burrows et al. (2011). The source is broad band, with photon emission from radio to the hard X-ray. By far, the overwhelming majority of the fluence comes in the form of X-ray emission from $\approx 0.1-100 \mathrm{keV}$ characterized by a flat spectral index $\Gamma \lesssim 2$ that is heavily obscured by absorption at low energies. ${ }^{1}$ The fact that the Fermi observatory did not report a detection of Sw $1644+57$ implies that the high energy spectrum is cutoff above Swift's Burst Alert Telescope's energy range. The source is coincident with the nucleus of its presumed host galaxy located at a luminosity distance of $1.6 \mathrm{Gpc}$.

The temporal properties of the event are bimodal. There were short-lived flares, the most powerful and energetic of which lasted $\sim 10^{3} \mathrm{~s}$ with an inferred isotropic X-ray luminosity $L_{X}^{\text {flare }} \sim 3 \times 10^{48} \mathrm{erg} / \mathrm{s}$. Over the next $\Delta t \sim 10^{6} \mathrm{~s}$, the behavior is more steady with $L_{X} \lesssim 10^{47}$ $\mathrm{erg} / \mathrm{s}$ and thus, the ratio of steady to flaring isotropic equivalent energy release is $\sim 30$. The inferred energy release during the period of steady activity $\Delta E_{X} \sim 10^{53}$ erg, close to the 1/10 rest mass of a typical star.

From here on, focus is directed primarily towards understanding the physical mechanisms behind the production of $\Delta E_{X}$ worth of $\mathrm{X}$-rays as it is responsible for $\sim 95 \%$ of the observed energy release. The fact that $\Delta E_{X}$ is close the gravitational binding energy of a typical star, at the inner-most circular orbit (ISCO) of a black hole suggests the following: (1) the event Sw J1644+57 is powered by black hole accretion (2) the accretion is radiatively efficient $(3)$ in conjunction with its transient nature, the event is likely due to the tidal disruption of $\mathrm{a} \sim 1 M_{\odot}$ star by a super-massive black hole.

\footnotetext{
${ }^{1}$ By definition, when $\nu F_{\nu}=$ a constant, $\Gamma=2$.
}

As envisioned by Rees (1988), a star becomes tidally disrupted during close approach to a super-massive black hole, once the orbital separation becomes comparable to the Roche radius. For a typical stellar radius $\sim R_{\odot}$, disruption can only occur for black holes that are $\lesssim 10^{8} M_{\odot}$. Black holes larger than $\sim 10^{8} M_{\odot}$ are unable to produce a tidal disruption event - unless larger stars are considered - since the star can be directly swallowed.

Even if the black hole mass $M_{\bullet} \sim 10^{8} M_{\odot}$, the average steady X-ray luminosity $L_{X}$ exceeds the Eddington Limit, $L_{\mathrm{Edd}}$, by a factor of $\sim 10$. If the Eddington Limit cannot be violated, the fluence of Sw J1644+57 cannot be linked to the source of fuel and energy in a straightforward manner. The flow may be out of hydrostatic balance, radiatively inefficient, geometrically and/or relativistically beamed (Bloom et al. 2011; Burrows et al. 2011).

The basic physical requirements for black hole accretion to overcome the Eddington Limit, while maintaining a high level of radiative efficiency, are outlined by Socrates \& Davis (2006; SD06). Given those requirements, they estimate that the spectral energy distribution of such flows are dominated by a flat $\Gamma \sim 2$ Comptonized X-ray power-law out to $\sim 100 \mathrm{keV}$. With the use of archival data from $X M M$, they show that the brightest Ultra-Luminous X-ray sources (ULXs) - the sources that clearly surpass the Eddington Limit for typical stellar mass black holes - are, in fact, characterized by flat spectral energy distributions with $\Gamma \sim 2$.

It therefore seems natural to describe the energy release mechanisms of $\mathrm{Sw} 1644+57$ as a scaled-up transient ULX i.e., an Ultra-Luminous X-ray event (ULX-E). In this Letter, the physical requirements, observational appearance and existing evidence of radiatively efficient super-Eddington accretion are given in $\S 2$. These principles are applied to Sw J1644+57 in $\S 3$. Observational tests and conclusions are briefly discussed in $\S 4$

2. RADIATIVELY EFFICIENT SUPER-EDDINGTON BLACK HOLE ACCRETION: REQUIREMENTS, APPEARANCE AND EXISTING EVIDENCE

2.1. Difficulty in overcoming the Eddington Limit 
For a black hole accretion flow, persistent emission above the Eddington Limit is even more prohibitive than say for example, a star. The Eddington Limit results from the condition of hydrostatic equilibrium

$$
\frac{\kappa}{c} \mathbf{F}=\nabla \phi
$$

in the limiting case where the gravitational acceleration $=\nabla \phi$ is balanced entirely by the radiation force, proportional to the radiative flux $\mathbf{F}$ and opacity $\kappa$. The expression above can be transformed into the Eddington Limit

$$
\begin{gathered}
\frac{\kappa}{c} L=\frac{\kappa}{c} \int_{\partial V} d \mathbf{A} \cdot \mathbf{F}=\int_{\partial V} d \mathbf{A} \cdot \nabla \phi=4 \pi G \int_{V} d^{3} x \rho \\
L=L_{\mathrm{Edd}}=\frac{4 \pi G M_{\mathrm{enc}} c}{\kappa} .
\end{gathered}
$$

Here, the opacity is taken to be a constant over the Gaussian surface $\partial V$, which bounds the volume $V$ of the flow. The mass of the disk is taken to be insignificant in comparison to the mass of the black hole so that $M_{\bullet}=M_{\mathrm{enc}}$ (see $\S$ II of Abramowicz et al. 1980). Note that the geometry of the surface $\partial V$ can be quite arbitrary, implying that the Eddington Limit is applicable for a disk, rather than just for an object with spherical symmetry. Given the mass of the black hole $M_{\bullet}, L_{\text {Edd }}$ is specified by the opacity, where $\kappa$ is set equal to the Thomson electronscattering opacity $\kappa_{e s}$.

In addition, once the accretion power exceeds Eddington, then steady radiatively efficient accretion is also hindered by the condition of radiative equilibrium. For standard (thin or slim) $\alpha$-disk accretion (Shakura \& Sunyaev 1973; Abramowicz et al. 1988), the condition is given by

$$
Q_{\mathrm{adv}}^{-}-\nabla \cdot \mathbf{F}=Q_{\mathrm{vis}}^{+} .
$$

The viscous dissipation rate $Q_{\text {vis }}^{+}$is balanced by a combination of radiative cooling perpendicular to the midplane and the inward advection of heat, quantified by $Q_{\mathrm{adv}}^{-}$.

SD06 note that the radius at which the heat generated by viscous dissipation is advected into the hole, rather than lost by radiative diffusion - mediated by the Thomson opacity $\kappa_{e s}$ - i.e., the trapping radius $R_{t r}$, for values of the accretion rate above $L_{\mathrm{Edd}} / c^{2}$, is given by

$$
R_{t r} \sim R_{g} \dot{m}
$$

where $R_{g}=G M_{\bullet} / c^{2}$ is the gravitational radius and $\dot{m}$ is the accretion rate in units of $L_{\mathrm{Edd}} / c^{2}$. For a flow with radiative efficiency $\epsilon$, the ISCO is located at $R_{\text {in }} \sim R_{g} / \epsilon$. It follows that as $L \rightarrow L_{\text {Edd }}$, then $R_{t r} \rightarrow R_{i n}$. At the Eddington Limit, the flow marginally maintains a high level of radiative efficiency. Since $R_{t r} \propto \dot{m}$, the net radiative efficiency decreases in proportion $\dot{m}^{-1}$ and the luminosity is pinned to the Eddington value - a result of the fact that gravitational power is $\propto 1 / R$.

\subsection{Physical requirements for surpassing the Eddington Limit}

Without addressing any deep theoretical mechanisms (turbulence, magnetic fields, reconnection, etc.,), it is possible to determine the necessary physical requirements for a black hole accretion flow to maintain a high level of radiative efficiency in the event that it persistently emits above the Eddington value.

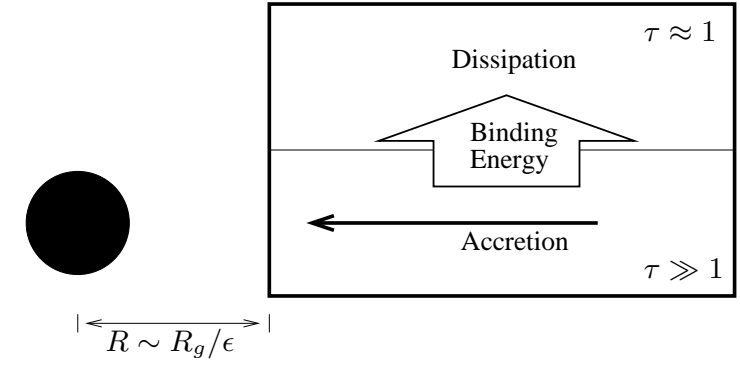

FIG. 1.- Geometry of flow. Not to scale.

In order to circumvent the "trapping problem," SD06 realized that there must be a form of vertical energy transport that separates binding energy from the mass of its origin, faster than radiative diffusion. An examination of eq. 3 reveals that the characteristic velocity at which thermal energy can be transported in the vertical direction by diffusion mediated by electron scattering is $v_{\text {diff }} \sim c / \tau$, where $\tau$ is the Thomson optical depth of the flow.

By construction, a corona is a region that possesses relatively small amounts of binding energy, but is the recipient of a disproportionate amount of it per unit time. As a result, the corona is heated to a temperature above the photospheric temperature of the optically thick binding energy reservoir.

If the trapping problem establishes the need for a corona, the departure from hydrostatic balance inferred from eq. 1, constrains its optical depth $\tau_{c}$. In the absence of other forces, the photosphere of the accretion flow cannot be in hydrostatic equilibrium and a radiation-driven wind is launched from the corona. In order to avoid the conversion of photon energy into outgoing mechanical power of the wind, the optical depth of corona $\tau_{c} \sim 1$ (SD06). If $\tau_{c} \gtrsim$ a few, photons becomes red-shifted as a result of adiabatic losses as they transfer their energy to matter. In such an event, the photon luminosity observed at infinity can be vanishingly small.

\subsection{Observational Appearance}

Powerful coronae universally appear in the spectral energy distribution of sub-Eddington accreting stellar and super-massive black holes. Though binding energy is stored in the ionic disk component, upon dissipation in the corona gravitational power is transferred to the electrons. Comptonization by seed photons results in a power law distribution of X-ray photons, indicative of a second order Fermi process (Kompane'ets 1956; Sunyaev \& Titarchuk 1980).

The spectral shape in the limit of un-saturated Comptonization by a thermal plasma is primarily determined by the Compton $y$-parameter given by $y=$ $\left(4 k_{B} T_{e} / m_{e} c^{2}\right) \tau_{c}$ in the limit that the corona is optically thin. A potential source of seed photons is the transitional region between the corona and the dense disk (Ross \& Fabian 1993; Ross et al. 1999; Ballantyne et al. 2001; Nayakshin et al. 2000; Nayakshin \& Kallman 2001). The cold dense disk is not a perfect reflector and down-ward Comptonized X-rays serve as a source of heat due to non-zero albedo. Above photon energies of $30 \mathrm{keV}$, Compton down-scattering dominates the albedo 
while at lower energies, photo-electric absorption mainly contributes to the albedo. These calculations show that for high values of ionizing flux, which is $\propto L_{c}$, fluorescence and absorption features in the X-ray spectrum persist, though at a highly suppressed level. The outgoing spectrum is roughly represented by a flat power law that extends to the cut-off energy given by $k_{B} T_{e}$. For AGN, the power law spans from tens of $\mathrm{eV}$ all the way to several hundreds of $\mathrm{keV} .^{2}$

\subsection{Existing Evidence: Bright ULXs}

The brightest ULXs with inferred bolometric luminosities $\sim 10 L_{\text {Edd }}$ for a stellar mass black hole are dominated by hard power-law X-rays. Such behavior is in line with the theoretical expectations outlined above.

In sub-Eddington black hole accretion flows that power black hole binaries (BHBs) and AGN, the fraction of energy dissipated in the corona $f$ is roughly given by the ratio of Comptonized to bolometric power. Table 1 shows that for BHBs, the ratio $L_{c} / L$ is correlated with $L / L_{\text {Edd }}$. For the "ULX state" (SD06), the black hole mass is not measured and is taken to have a characteristic value of $10 M_{\odot}$.

Furthermore, Grimm et al. (2003) construct the combined luminosity function of local star-forming galaxies. When they restrict their study to high mass X-ray binaries (HMXBs; companion mass $>5 M_{\odot}$ ), they find that the luminosity function is well-described by a single power-law up to (un-extrapolated) luminosities of $3 \times 10^{40} \mathrm{erg} / \mathrm{s}$ i.e., 30 times the Eddington Limit for a $10 M_{\odot}$ black hole (see their figure 5). If these spectra were modeled and extrapolated to higher energies (100 $\mathrm{keV}$ ), then their luminosity would extend to $\sim 10^{41} \mathrm{erg} / \mathrm{s}$. Both properties of their luminosity function simultaneously dictate that super-Eddington accretion is at work and that it is radiatively efficient.

If intermediate mass black holes (IMBHs) are responsible for the population of extra-galactic HMXBs above the characteristic Eddington Limit, then one would expect a break in the luminosity function. It is unreasonable to expect that IMBHs are members of binary systems in the same number as stellar mass black holes. If the high end of the HMXB luminosity function signals superEddington luminosities from radiatively in-efficient accretion, then luminosity function would exhibit a break above $L_{\text {Edd }}$. Similarly, a beamed-jet explanation for the high end of the HMXB luminosity function would produce a break in the luminosity function. The fact that the luminosity function is a power-law is most easily interpreted by realizing that the central sources of HMXBs are wind-fed and the rate at which high mass stars donate mass to their compact companions is set by stellar evolution.

The fact that the spectral energy distributions of the bright ULXs possess the same properties - from basic theoretical arguments and observational expectations as that of super-Eddington radiatively efficient accretion

\footnotetext{
2 The author thanks J. Goodman and J. Gunn for pointing out that the observed spectrum, in conjunction with the criteria that $\tau_{c} \sim 1$, implies that thermal disk photons cannot be responsible for the seed photons for bright ULXs - in contradiction to the assertions of SD06. In the Appendix it is further shown that thermal disk emission cannot escape into the corona as it is trapped inward to due to advection.
}

TABLE 1

Spectral Classification of Luminous Black Hole Binaries Based on Coronal Behavior

\begin{tabular}{ccc}
\hline \hline Spectral State & $L / L_{\mathrm{Edd}}$ & $L_{c} /\left(L-L_{c}\right)$ \\
\hline High/Soft & 0.1 & 0.1 \\
Very High & 1.0 & 1.0 \\
ULX & 10.0 & 10.0 \\
\hline
\end{tabular}

${ }^{\mathrm{a}}$ The values listed above are approximate. Fractional coronal power is correlated with bolometric luminosity.

flows is suggestive. In addition, the luminosity function of the extra-galactic HMXBs reinforces the notion that bright ULXs represent a mode of black hole accretion that is both super-Eddington and radiatively efficient.

\section{MODEL FOR SW J1644+57: AN ULTRA-LUMINOUS X-RAY EVENT}

In its observational appearance as seen through its spectral energy distribution, Sw $1644+57$ can be thought of as a scaled-up bright ULX. Again, the bright ULXs represent a class of stellar mass sources objects that persistently emit well above the Eddington Limit with high radiative efficiency. Therefore, it seems natural to construct an accretion model of Sw J1644+57 that resembles those of bright ULX, multiplied by a factor of $\sim 10^{6}-10^{7}$ in mass.

Rather than steady feeding from a companion star, the source of fuel results from the tidal disruption of an ordinary star (Rees 1988). There has been recent theoretical progress on this topic (Rosswog \& Ramirez-Ruiz 2009), yet the resulting spatial distribution of the debris is remains uncertain. Assume that $\sim 1 M_{\odot}$ of stellar gas is placed in a circular orbit at radius $R_{0}$ that is some multiple $\beta$ of the ISCO located at $R_{g} / \epsilon$. Within a dynamical time, the disk becomes viscous and - for lack of a better choice - the viscosity can be approximated by the usual $\nu \sim \alpha c_{s} H$ (Shakura \& Sunyaev 1973). The viscous time $t_{\nu}$ determines the characteristic duration of the event. Since the radiation cooling time is long in comparison to the viscous time, the flow is likely to be geometrically thick i.e., the vertical scale height $H \sim R$ with sound speed $c_{s}^{2} \sim G M_{\bullet} / R$. In terms of these parameters, the viscous time is given by

$$
\begin{aligned}
& t_{\nu}=R_{0}^{2} / \nu \sim \frac{\beta^{2} R_{g}^{2}}{\epsilon^{2} \alpha c_{s} H} \sim \frac{\beta^{3 / 2} R_{g}}{\epsilon^{3 / 2} \alpha c} \\
& t_{\nu} \sim 3 \times 10^{6} \frac{\beta_{10}^{3 / 2} M_{\bullet}, 8}{\epsilon_{0.1}^{3 / 2} \alpha_{0.1}} \mathrm{~s} .
\end{aligned}
$$

Reasonable changes in $\epsilon$ and $\beta$ lead to comparable values of $t_{\nu}$ for a variation of $\sim 10$ in $M_{\bullet}$. Reported variability timescales of $\sim 100 \mathrm{~s}$ indicate that $M_{\bullet} \sim 10^{7} M_{\odot}$. The size of the host galaxy further supports the "small" black hole hypothesis.

If $\sim 1 M_{\odot}$ worth of material is swallowed with radiative efficiency $\epsilon \sim 0.2$, then the event can support a luminosity $L \sim 10^{47} \mathrm{erg} / \mathrm{s}$ for $3 \times 10^{6} \mathrm{~s}$. If $M_{\bullet} \sim 10^{7} M_{\odot}$, then $L / L_{\text {Edd }}$ is consistent with the high end of Eddington ratios for extra-galactic HMXBs.

Since $L>L_{\mathrm{Edd}}$, hydrostatic balance is broken in the absence of other forces (e.g., magnetic fields anchored 
deep in the cold dense disk SD06). A Compton radiationdriven wind develops and even for a small black hole with $M_{\bullet} \sim 10^{7} M_{\odot}$, the total compactness $\ell \equiv \epsilon L / L_{\text {Edd }}$ is only $\sim 10$. Under these conditions, a Compton-driven wind is inefficient at converting the input photon luminosity into bulk wind power (Madau \& Thompson 2000). On energetic grounds, a heavily Comptonized medium is unable to efficiently launch a powerful wind since, by construction, the electrons are donating energy to the photons as they attempt to they gain momentum from them (Phinney 1982).

Along with the low coronal optical depth $\tau_{c}$, which implies that adiabatic losses are not catastrophic, it seems reasonable that a $\sim 10^{7} M_{\odot}$ black hole can accrete a stellar mass in $\sim 10^{6} \mathrm{~s}$ with high radiative efficiency as long as a fraction $f$ of the binding energy dissipated in the corona is $\approx 1$. The observed shape of the photon spectrum resembles those of the brightest ULXs i.e., a flat power-law in X-rays.

\section{QUESTIONS, PREDICTIONS AND CONCLUSIONS}

The central energy source of $\mathrm{Sw} \mathrm{J} 1644+57$ is heavily obscured. It is almost a certainty that, due to energy conservation, all of dust-obscured photon power will be re-emitted in the IR wavebands at some later time. If the source is an isotropic emitter, such as the ULX-E model presented here, the total energy emitted in the IR is likely to exceed that of a beamed jet model. If a sizeable fraction of $10^{53} \mathrm{ergs}$ is absorbed by dust, then the reprocessed IR emission will easily out-shine the host as long as the thermal relaxation time of the dusty medium does not exceed several decades.

As stellar debris drains into the black hole, the rate of accretion drops, as does $\tau$ of the dense disk and the bulk of the flow is eventually able to radiate efficiently. SubEddington AGN and BHBs possess spectra that show strong thermal emission. Once the accretion power drops below the Eddington Limit, the characteristic energy of the disk photons $\sim 50 \mathrm{eV}$ for $M_{\bullet} \sim 10^{7} M_{\odot}$. There may be enough optical-UV power in the thermal component at later times so that the central source out-shines the host galaxy.

Epochs of intense and highly variable flaring X-ray power as well as radio emission (Giannios \& Metzger 2011), are most likely due to the production of a jet at early times. Swift's wide-field BAT instrument was triggered by these bright flaring events, rather than the relatively dim persistent radiative emission responsible for the bulk of the energy release $\Delta E_{X}$. If the jet openingangle is sufficiently small, then the rate of tidal disruption events are in line with theoretical expectations (Bloom et al. 2011; Burrows et al. 2011; see Magorrian \& Tremaine 1999) .

Even for sub-Eddington accretion flows, the nature of the transport mechanism that energizes the corona has not been identified for thirty-five years (e.g., Shapiro et al. 1976). Possibilities include magnetic buoyancy (Galeev et al. 1979), wave excitation and turbulence (Socrates et al. 2004; Thompson 2006). The high compactness of black hole and neutron star accretion flows in comparison to other astrophysical systems may explain why their inferred value of $f$ is so high (Goodman \& Uzdensky; Socrates 2010). Nevertheless, the distance between a robust and testable theory that quantifies the fraction $f$ of binding energy dissipated in the corona and our current understanding seems far. The possibility that $\mathrm{Sw} \mathrm{J} 1644+57$ is powered by coronal energization reinforces the general desire to identify the physical mechanisms that power Comptonized radiation of relativistic accretion flows.

It is a pleasure to thank J. Miradla-Escude, E. Ramirez-Ruiz and R. Sunyaev for helpful conversations as well as D. Giannios and B. Metzger for comments on the manuscript. Support form a John Bahcall Fellowship awarded by the Institute for Advanced Study, Princeton is acknowledged.

\section{THE DENSE DISK MUST BE ADVECTION DOMINATED}

Let $t_{\mathrm{adv}} \equiv R / v_{R}$ and $t_{\mathrm{diff}} \equiv \tau H / c$ where

$$
v_{R}=\frac{\dot{M}}{4 \pi R H \rho}=\frac{\dot{M} \kappa}{4 \pi R \tau}
$$

and $\dot{M}$ is the accretion rate. Compute the ratio

$$
\frac{t_{\mathrm{adv}}}{t_{\mathrm{diff}}}=\frac{4 \pi R^{2} \tau}{\dot{M} \kappa} \frac{c}{\tau H}=\frac{4 \pi R \epsilon c^{2}}{L \kappa} .
$$

Most of the energy is liberated near the ISCO, located at $R \sim R_{g} / \epsilon=G M_{\bullet} / \epsilon c^{2}$ and at that location

$$
\frac{t_{\mathrm{adv}}}{t_{\mathrm{diff}}}=\frac{R}{R_{g}} \frac{\epsilon}{L / L_{\mathrm{Edd}}} \sim \frac{L_{\mathrm{Edd}}}{L} .
$$

The cool dense disk it too optically thick to cool when $L \gg L_{\text {Edd }}$ even if the gravitational power it generates is dissipated exterior to it, in the adjacent corona. It follows that due to the long diffusion time, the disk cannot serve as a source of seed photons for the super-Eddington corona, in contradiction to the claim of SD06. In other words, the fraction of gravitational power dissipated in dense the disk $=(1-f)$ cannot escape via radiative diffusion. Once the accretion power becomes super-Eddington, the optically thick thermal emission component generated near the ISCO disappears and is swallowed into the black hole. Only the fraction $f$ of the binding energy deposited in the corona is available for the production of escaping radiation. In order for the flow to maintain a high level of radiative efficiency above the Eddington Limit, $f \approx 1$.

\section{REFERENCES}


Ballantyne, D. R., Ross, R. R., \& Fabian, A. C. 2001, MNRAS, 327,10

Bloom, J. S., et al. 2011, arXiv:1104.3257

Burrows, D. N., et al. 2011, arXiv:1104.4787

Galeev, A. A., Rosner, R., \& Vaiana, G. S. 1979, ApJ, 229, 318

Giannios, D., \& Metzger, B. D. 2011, arXiv:1102.1429

Goodman, J., \& Uzdensky, D. 2008 ApJ, 688, 555

Galeev, A. A., Rosner, R., \& Vaiana, G. S. 1979, ApJ, 229, 318

Grimm, H.-J., Gilfanov, M., \& Sunyaev, R. 2003, MNRAS, 339, 793

Levan, A. J., et al. 2011, arXiv:1104.3356

Madau, P., \& Thompson, C. 2000, ApJ, 534, 239

Magorrian, J., \& Tremaine, S. 1999, MNRAS, 309, 447

Nayakshin, S., Kazanas, D., \& Kallman, T. R. 2000, ApJ, 537, 833

Nayakshin, S., \& Kallman, T. R. 2001, ApJ, 546, 406
Phinney, E. S. 1982, MNRAS, 198, 1109

Rees, M. J. 1988, Nature, 333, 523

Ross, R. R., \& Fabian, A. C. 1993, MNRAS, 261, 74

Ross, R. R., Fabian, A. C., \& Young, A. J. 1999, MNRAS, 306, 461

Ramirez-Ruiz, E., \& Rosswog, S. 2009, ApJ, 697, L77

Shakura, N. I., \& Sunyaev, R. A. 1973, A\&A, 24, 337

Socrates, A. 2010 ApJ, 719, 784

Socrates, A., Davis, S.W., \& Blaes, O.M. 2004 ApJ, 601, 405

Socrates, A., \& Davis, S.W. 2006, ApJ, 651, 1059

Sunyaev, R. A., \& Titarchuk, L. G. 1980, A\&A, 86, 121

Thompson, C. 2006, ApJ, 651, 333 